\title{
Huddling relieves myocardial glycogen synthesis of Brandt's voles in mild cold environment
}

\author{
Zhe Wang \\ Qufu Normal University \\ Jun-jie Mou \\ Qufu Normal University \\ Jin-hui Xu ( $\nabla$ xujinhui@qfnu.edu.cn ) \\ https://orcid.org/0000-0002-5550-1764 \\ Chuan-li Wang \\ Qufu Normal University \\ Wei-mei Huang \\ Qufu Normal University \\ Hui-liang Xue \\ Qufu Normal University \\ Ming Wu \\ Qufu Normal University \\ Lei Chen \\ Qufu Normal University \\ Lai-xiang Xu \\ Qufu Normal University
}

\section{Research}

Keywords: huddling, low temperature, heart, glycogenosomes, glycogen synthetase, glycogen phosphorylase

Posted Date: May 24th, 2020

DOI: https://doi.org/10.21203/rs.3.rs-30447/v1

License: (c) (1) This work is licensed under a Creative Commons Attribution 4.0 International License. Read Full License 


\section{mild cold environment}

3 Zhe Wang ${ }^{\#}$, Jun-jie Moü, Jin-hui Xu${ }^{\# *}$, Chuan-li Wang, Wei-mei Huang, Hui-liang Xue, Ming Wu,

4 Lei Chen, Lai-xiang $\mathrm{Xu}^{*}$

5 (College of Life Sciences, Qufu Normal University, 273165, Qufu, Shandong, China)

$6 \quad$ \# Zhe Wang, Jun-jie Mou and Jin-hui Xu contributed equally to this work.

7 *Correspondence and requests for materials should be addressed to J.-H.X

8 (email: xujinhui@qfnu.edu.cn ) and X.-L.X (email: xulx@qfnu.edu.cn )

9 
Background:Small mammals have limited glucose use and limited glycogen accumulation during hypothermia. Huddling is a highly evolved cooperative behavioral strategy in social mammals, allowing adaptation to environmental cooling. As yet, however, is not clear whether this behavior affects the utilization of glycogen in cold environments. Here, we studied the effect of huddling on myocardial glycogen content in Brandt's voles (Lasiopodomys brandtii) under a mild cold environment $\left(15^{\circ} \mathrm{C}\right)$.

Results:Results showed that (1) Compared to the control $\left(22^{\circ} \mathrm{C}\right)$ group $(\mathrm{CON})$, the number of glycogenosomes more than tripled in the cool separated group (CS) in both males and females; whereas the number of glycogenosomes increased in females but was maintained in males in the cool huddling group (CH). (2) The ratio of glycogen synthetase phosphorylation showed a similar trend as the change in glycogenosome number in the three treatment groups in both males and females. (3) Protein expression of glycogen phosphorylase remained stable in males in the three treatment groups but increased in CS group females compared with the CON group.

Conclusion:These results indicate that huddling in voles alleviated the increase in myocardial glycogen content caused by the increase of glycogen synthesis under cool environments.

Keywords: huddling, low temperature, heart, glycogenosomes, glycogen synthetase, glycogen phosphorylase

\section{Introduction}

Low temperature is a stress stimulus for mammals, especially for small mammals as their energy requirements are high due to the large surface area to volume ratio. Moreover, when environmental stressors persist for prolonged periods, small animal tissues and organs are more vulnerable to the impact of external environmental temperature [1]. Altered carbohydrate metabolism during hypothermia in mammals is accompanied by abnormalities in glucose metabolism [2-4]. For example, in rats [5, 6] and rabbits [7], metabolism of both endogenously and exogenously administered glucose is substantially reduced during hypothermia. Hypothermia can lead to a slowed heart rate, decreased blood flow output, and decreased myocardial contraction and relaxation function [8-11]. As above, the cardiac muscle of small mammals is more susceptible to low external temperatures. In rats, for example, exposure to only $4 \mathrm{~h}$ of cold temperature $\left(15^{\circ} \mathrm{C}\right)$ can lead to an increase in myocardial glycogen content [12], suggesting that the effects of hypothermia on cardiac muscle may involve the balance between glycogen synthesis and degradation.

Glycogen synthase (GS), a key enzyme for synthesis, polymerizes UDP-glucose to form glycogen granules, with phosphorylated GS (P-GS) being its active state [13-15]. Glycogen phosphorylase (GYPL) is a rate-limiting enzyme that breaks down glycogen granules to glucose $[16,17]$. Research on hibernating Daurian ground squirrels (Spermophilus dauricus) has shown that the increase in glycogen content in skeletal muscle in winter is mainly due to the maintenance of P-GS and decrease in GYPL protein expression [15]. Thus, studies on the above factors could help reveal the mechanism related to changes in myocardial glycogen content under cool environments.

Huddling is a social thermoregulatory behavior, defined as the active aggregation of nestled animals. It is a cooperative group behavior, permitting individuals involved in social

51 thermoregulation to minimize heat loss and thereby lower energy expenditure, possibly allowing 
reallocation of saved energy to other functions [1, 18]. It is commonly exhibited in small mammals and birds to reduce heat and energy loss under cold environments [19-22]. Research has shown that many mammals, such as degu (Octodon degus), Damaraland mole-rat (Cryptomys damarensis), and Natal mole-rat (C. hottentotus natalensis), huddle when the ambient temperature is lower than $15-20^{\circ} \mathrm{C}$, with an energy saving of up to $30 \%$ [23, 24]. Research on Eastern pygmy possums (Cercartetus nanus) has shown that huddling in mild low temperatures $\left(14{ }^{\circ} \mathrm{C}\right)$ can reduce energy consumption by up to $50 \%$ [25]. The benefits of huddling in energy conservation [23, 26], local environmental heating [27], and survival [28] have also been studied in several species. Overall, huddling individuals exhibit increased survival, lower food intake, decreased body mass loss, increased growth rate, and/or more constant body temperature, and reduced metabolic rate [1]. To date, previous studies have primarily focused on morphological and physiological changes in animal bodies under various temperatures. However, no studies have reported on changes in myocardial glycogen in mammals under different temperatures.

Brandt's voles (Lasiopodomys brandtii) are small non-hibernating herbivorous rodents widely distributed in the Inner Mongolian grasslands of Northern China, dry steppe zone of Mongolia, and southeast Baikal region of Russia. They are highly socialized animals that huddle in winter as an adaptation to their harsh habitats [20]. Studies have shown that mild cooling can significantly change the level of oxidative stress in the cardiac muscle of Brandt's voles [29]. Furthermore, their metabolic rate and thermogenic capacity decrease but activity increases compared with separated individuals under low temperatures, suggesting that huddling is a good strategy for small mammals to cope with cold environments [19]. Glycogen is one of the most important energy supply substances in muscles. However, the role of myocardial glycogen in adaptive huddling has not yet been reported. Therefore, we hypothesized that a cool environment could cause an increase in myocardial glycogen content in Brandt's voles. We also hypothesized that huddling could effectively alleviate this change. To test these hypotheses, we observed the ultrastructure of cardiac muscle in huddling and individual (separated) Brandt's voles under mild temperature differences (normal: $22{ }^{\circ} \mathrm{C}$; cool: $15{ }^{\circ} \mathrm{C}$ ) in autumn. We also determined the protein expression levels of glycogen synthesis and degradation-related signals. We further explored the molecular mechanism related to the effects of a mild cold environment and huddling on changes in myocardial glycogen content.

\section{Materials and Methods}

\section{Ethics statement}

All procedures followed the Laboratory Animal Guidelines for the Ethical Review of Animal Welfare (GB/T 35892-2018) and were approved by the Animal Care and Use Committee of Qufu Normal University (Permit Number: dwsc 2019012).

\section{Animals and groups}

In early September 2019, Brandt's voles were captured by live trapping from an enclosure at the Inner Mongolia Grassland Animal Ecology Research Station $(60 \times 80 \mathrm{~m})$, located in the Maodeng Pasture $\left(44^{\circ} 11^{\prime} \mathrm{N}, 116^{\circ} 27^{\prime} \mathrm{E}\right.$; $1100 \mathrm{~m}$ in elevation) of Xilinhot, Inner Mongolia, China. Voles were acclimated under laboratory conditions for two weeks. They were housed individually in cages (28 $\times 18 \times 12 \mathrm{~cm}$ ) at an ambient temperature of $22 \pm 2{ }^{\circ} \mathrm{C}$ and relative humidity of $55 \% \pm 5 \%$ under a 

Experimental Animal Breeding Co., Ltd., China) and water were provided ad libitum and wood shavings were used as bedding.

Based on body weight and degree of wear on the upper molars, a total of 24 male (28-50 g, average $38 \mathrm{~g}$ ) and 24 female adult voles (27-54 g, average $33 \mathrm{~g}$ ) were randomly divided into three groups, respectively. Voles in the control groups (CON) of each sex were housed individually in cages at an ambient temperature of $22 \pm 2{ }^{\circ} \mathrm{C}$, but with a changed light regime to a short day pattern ( $8 \mathrm{~h}: 16 \mathrm{~h}$ lightdark cycle; light on from 08:00 to 16:00). Two groups of each sex were transferred to a cool cabinet under different grouping conditions (huddling and separated). Voles in the cool separated groups (CS) were housed individually in cages, whereas voles in the cool huddling groups $(\mathrm{CH})$ were housed together in a cage (two males and two females). Group size (four voles in each cage) ensured most animals remained inactive in a huddle (Sukhchuluun et al., 2018). The temperature in the cabinet was set to $15{ }^{\circ} \mathrm{C}$ and the light regime was as same as the CON group. Animal treatment started in late September and lasted eight weeks.

\section{Sample preparation}

All animals were anaesthetized with $50 \mathrm{mg} \mathrm{kg}^{-1}$ sodium pentobarbital between 08:00 and 11:00 on the last day of the experimental period [19, 30]. After the rapid removal of cardiac muscle, portions of the ventricles were immediately cut off and fixed in glutaraldehyde. The rest of the cardiac muscle was frozen in liquid nitrogen and stored at $-80^{\circ} \mathrm{C}$. Specimens were fixed in $1 \%$ osmium tetroxide in the same buffer, dehydrated with a graded series of ethanol, and embedded in epoxy resin. After surgical intervention, the animals were sacrificed with an overdose injection of sodium pentobarbital. All procedures were carried out in accordance with the approved guidelines.

\section{Transmission electron microscopy (TEM)}

The cardiac muscle samples were cut into blocks and immersed in $3 \%$ glutaraldehyde-paraformaldehyde. The blocks were then dehydrated in a graded series of ethanol and embedded in epoxy resin, with TEM then performed as described previously [31]. A semithin section was applied to tissue samples, and after methylene blue staining [32], sections were adjusted under the microscope and sliced with an ultramicrotome (LKB-NOVA, USA). The ultrathin sections were double stained with Reynolds' lead citrate and ethanolic uranyl acetate [33] and then examined via TEM (Hitachi, HT7800, Japan). Images were processed with NIH Image-Pro Plus 6.0. Images were analyzed using the measurement tools provided by the software. Glycogenosome densities were determined within a defined region $\left(4 \mu \mathrm{m}^{2}\right.$ area $)$ at a minimum of three locations within an image taken at $25000 \times$ magnification.

\section{Western blotting}

Total protein was extracted from the tissues and solubilized in sample buffer (100 mM Tris, $\mathrm{pH}$ $6.8,5 \% 2-\beta$-mercaptoethanol, $5 \%$ glycerol, $4 \%$ SDS, and bromophenol blue), with the extracts of cardiac protein then resolved via SDS-PAGE $(10 \%$ Laemmli gel with an acrylamide/bisacrylamide ratio of 29:1 and 98\% 2,2,2-trichloroethanol (Aladdin, JI522028, China)). To study protein expression in different tissues, we used total protein content as a reference. After electrophoresis, the gel was irradiated on the UV platform of the electrophoresis gel imaging analysis system (Bio-Rad, California, USA) for $5 \mathrm{~min}$, after which the signal was 
collected. As described previously [34, 35], the original image captured with no gain was stored. After that, the fluorescence intensity of each lane (after removal of background fluorescence intensity) was determined with Image-Pro Plus 6.0, which as an internal reference, to correct the fluorescence intensity of the target protein. The proteins were then electrically transferred to polyvinylidene fluoride (PVDF) membranes $(0.45-\mu \mathrm{m}$ pore size) using a Bio-Rad wet transfer apparatus. The blotted membranes were blocked with 5\% skimmed milk powder in Tris-buffered saline (TBS; $150 \mathrm{mM} \mathrm{NaCl}, 50 \mathrm{mM}$ Tris-HCl, $\mathrm{pH}$ 7.5) and incubated with rabbit anti-glycogen phosphorylase (1:1 000, \#ab198268, Abcam, Cambridge, UK), rabbit anti-glycogen synthase (1:1 000, \#3886, Cell Signaling Technology CST, Danvers, MA, USA), and rabbit anti-phospho glycogen synthase $\left(1: 1000, \# 3891\right.$, CST) in TBS containing $0.1 \%$ BSA at $4{ }^{\circ} \mathrm{C}$ overnight. The membranes were then incubated with IRDye $800 \mathrm{CW}$ goat anti-rabbit secondary antibodies (1:5 000, \#31460, Thermo Fisher Scientific, Rockford, IL, USA) for $90 \mathrm{~min}$ at room temperature and visualized with an Odyssey scanner (Bio-Rad, CA, USA). Quantification of blots was performed using NIH Image-Pro Plus 6.0.

\section{Statistical analyses}

The normality of data and homogeneity of variance were tested by Shapiro-Wilk and Levene tests, respectively. All data exhibited normal distribution and homogeneous variance. Double-factor variance analysis (two-way analysis of variance (ANOVA)) was used to compare differences between treatment and sex. Results were significant at $P<0.05$. Data are expressed as mean \pm standard deviation (Mean \pm SD). All statistical analyses were conducted using SPSS 19.0.

\section{Results}

\section{Ultrastructural changes in number of glycogenosomes}

The glycogenosome clusters were observed, with each glycogenosome showing a diameter of $\sim 30$ $\mathrm{nm}$. Most glycogenosomes were distributed between the muscle filaments, with a small number distributed around the mitochondria (Fig. 1).

\section{INSERT FIGURE 1 HERE}

Figure 2a shows the distribution of glycogenosomes at low magnification. In the CS group, the number of glycogenosomes was more than triple that in the $\mathrm{CON}$ and $\mathrm{CH}$ groups $(P<0.05)$. In addition, the number was significantly higher $(P<0.05)$ in females than in males (Fig. 2b).

\section{INSERT FIGURE 2 HERE}

\section{Changes in protein expression of glycogen synthesis-related proteins}

The GS and P-GS concentrations were detected by western blot analysis, as shown in Fig. 3. Representative polyacrylamide gels of total protein are shown in Fig. $3 b$.

The relative protein expression levels of GS and P-GS showed the different trends among the three treatment groups. Specifically, the protein expression levels of GS in the CS groups were lower than the levels in the $\mathrm{CH}$ and CON groups, whereas protein expression levels of P-GS in the $\mathrm{CH}$ and $\mathrm{CS}$ groups were higher than levels in the CON group $(P<0.05)$. Levels of GS and P-GS were also higher $(P<0.05)$ in females than in males (Fig. 3c and d). 
The P-GS to GS ratio is one of the most direct indicators of glycogen synthesis. Here, the ratio showed the trends among the three treatment groups as $\mathrm{CON}<\mathrm{CH}<\mathrm{CS}(P<0.05)$. The ratio was also higher $(P<0.05)$ in females than in males (Fig. 3e).

\section{INSERT FIGURE 3 HERE}

\section{Changes in protein expression of glycogen decomposition-related proteins}

The content of GYPL was detected by western blot analysis, as shown in Fig. 4. Representative polyacrylamide gels of total protein are shown in Fig. $4 \mathrm{~b}$.

The relative protein expression of GYPL showed a slight change among the three treatment groups. Specifically, levels were higher in CS group females than in CON group females (Fig. 4c).

\section{INSERT FIGURE 4 HERE}

\section{Discussion}

We studied the effects of cool environment on the number of cardiac glycogenosomes in huddling Brandt's voles, as well as the mechanism related to the regulation of glycogenosome number. An obvious increase in the number of myocardial glycogenosomes was observed in both CS males and females. In addition, this number remained stable in males but increased in females in the $\mathrm{CH}$ groups compared with that in the CON groups. The P-GS to GS ratio was highest in the CS group in both males and females. GYPL protein expression only showed a slight change among the three treatment groups.

One of the most important findings of this study is the ultrastructural observation of a significant increase in the number of cardiac glycogenosomes in the CS group. Changes in myocardial glycogen in mammals during long-term cool environment exposure have not been reported previously, although our results are consistent with those of myocardium under short-term hypothermia and skeletal muscle under long-term hypothermia, as the major types of muscle fibers in ventricles are similar to those in soleus muscle [36]. Research on rats has shown that glycogen content in the myocardium is significantly increased after only $4 \mathrm{~h}$ of exposure to $15^{\circ} \mathrm{C}$ [12]. Furthermore, Daurian ground squirrels experience an increase in glycogen concentrations in in soleus muscle after two months of low temperature exposure in winter [15]. Excessive glycogen accumulation in the heart can lead to degenerative changes such as arrhythmia, cardiac hypertrophy, and hypotonia [37]. In this study, glycogen content in the myocardium of the CS group was significantly higher than that of the CON group. This indicates that hypothermia may cause significant degenerative damage to the myocardium of small mammals and may involve disrupting the balance between glycogen synthesis and decomposition. As the active state of GS, P-GS is an important indicator of glycogen synthesis [38]. Furthermore, GYPL is a rate-limiting enzyme that breaks down glycogen granules to glucose $[16,17]$. In males, the protein expression of GYPL in the CS group was maintained at the same level as observed in the CON group, whereas the phosphorylation ratio of GS was significantly increased, suggesting that high glycogen synthesis may be one of the main reasons for the increase in glycogen content. In females, the protein expression of GYPL and the phosphorylation ratio of GS increased significantly in the CS group, but the increment between the two groups was significantly different compared with the CON group. This indicated that the increase in glycogen synthesis 
was higher than that of glycogen decomposition, which may be the reason why glycogen content increased at this time. In general, the increase in glycogen synthesis may be a key factor for the increase in myocardial glycogen content observed in the CS group in both male and female voles.

Surprisingly, compared with the CON group, the myocardial glycogen content and phosphorylation rate of GS in $\mathrm{CH}$ male voles remained unchanged. Compared with the $\mathrm{CON}$ group, the protein expression levels of GYPL also remained unchanged in the hearts of both male and female voles in the $\mathrm{CH}$ group, indicating that maintenance of glycogen synthesis and decomposition may have led to maintenance of glycogen content in the $\mathrm{CH}$ group. This suggests that the effect of low temperature on glycogen synthesis can be significantly alleviated by huddling behavior. In addition, the myocardial glycogen content and phosphorylation rate of GS in $\mathrm{CH}$ female voles was significantly higher than that in the CON group, although the degree of increase was much lower than that found in the CS group. This suggests that huddling behavior partially alleviated the effect of low temperature on glycogen synthesis in female voles. In general, huddling behavior completely or partially alleviated the increase in glycogen content caused by the increase in glycogen synthesis in the myocardium of voles following cold environment exposure. Normal glycogen metabolism is the basis of exercise in mammals [39, 40]. Earlier studies on Brandt's voles showed that activity is higher in huddling groups than separated groups under cool environments [19]. Thus, we speculated that the results that the level of glycogen metabolism in the myocardium of $\mathrm{CH}$ group was closer to the level of CON group than that of CS group might be one of the reasons.

Interestingly, the biggest difference between the sexes was in myocardial glycogen content and phosphorylation rate of GS, which increased in $\mathrm{CH}$ females but remained unchanged in $\mathrm{CH}$ males compared to the CON groups. This may be because the average weight of males selected in this study was larger than that of females. Higher body weight may mean more fat and substance storage and therefore possible higher tolerance to lower temperatures.

In summary, we explored the regulatory mechanism related to the balance between glycogen synthesis and degradation on the number in myocardial glycogenosomes of huddling and separated Brandt's voles under cool environments. Results showed that a cool environment led to an increase in myocardial glycogen content in voles, which could be alleviated by huddling behavior, and may be a good consequence of the collective overwintering behavior of socialized animals. An increase in glycogen synthesis is a common mechanism for changes in myocardial glycogen level. The differences in glycogen content and related mechanism between the sexes mainly existed in the $\mathrm{CH}$ group, which could be impacted by differences in sample size between the two groups. In general, huddling behavior in voles alleviated the increase in myocardial glycogen content caused by the increase in glycogen synthesis.

\section{Acknowledgements}

Not applicable

\section{Author Contributions}

Z.W., J.-H.X., and L.-X.X. conceived and designed the research; Z.W., J.-H.X., J.-J.M., C.-L.W. 
and W.-M.H. performed the experiments; Z.W. and J.-J.M analyzed the data; Z.W. interpreted the experimental results; Z.W. and J.-J.M. prepared the figures; Z.W. and J.-H.X. drafted the manuscript; H.-L.X., M.W. and C. L. provided experimental guidance and suggestions for revision; J.-H.X., Z.W., and L.-X.X. edited the manuscript and approved the final version.

\section{Funding}

This work was supported by funds from the National Natural Science Foundation of China (No. $31770455,31670385,31570377$ )

\section{Availability of data and materials}

The datasets and original images are included in the supplementary documents.

\section{Ethics approval and consent to participate}

All procedures followed the Laboratory Animal Guidelines for the Ethical Review of Animal Welfare (GB/T 35892-2018) and were approved by the Animal Care and Use Committee of Qufu Normal University (Permit Number: dwsc 2019012).

\section{Consent for publication}

Not applicable.

\section{Competing interests}

The authors declare that they have no competing interests.

\section{Legend of figures}

Fig. 1 Ultrastructural distribution of myocardial glycogenosomes in Brandt's voles.

Arrow indicates glycogenosome. Z-zone ( $\mathrm{Z}$ ) and muscle filaments (see asterisk) are well arranged. Scale bar $=0.5 \mu \mathrm{m}$.

Fig. 2 Changes in number of myocardial glycogenosomes in Brandt's voles.

(a) Myocardial glycogenosomes in three treatment groups. Arrow indicates glycogenosome. Scale bar $=1 \mu \mathrm{m}$. (b) Bar graph depicting changes in number of glycogenosomes. Values are mean \pm SD. Six figures were analyzed in each sample; eight samples were analyzed in each group. CON, control group; $\mathrm{CH}$, cool huddling group; CS, cool separated group. Different letters identify statistically significant differences among temperature treatment groups $(P<0.05)$. ${ }^{*}, P<0.05$ significant differences between males and females.

Fig. 3 Changes in protein expression levels of glycogen synthesis-related factors in cardiac muscle of Brandt's voles.

(a) Representative immunoblots of GS and P-GS in cardiac muscle. (b) Representative polyacrylamide gel of total protein. (c) Relative protein expression of GS. (d) Relative protein expression of P-GS. (e) Ratio of P-GS to GS. Values are mean \pm SD. $n=8$. CON, control group; $\mathrm{CH}$, cool huddling group; CS, cool separated group. Different letters identify statistically significant differences among temperature treatment groups $(P<0.05) .{ }^{*}, P<0.05$ significant differences between males and females. 
Fig. 4 Changes in protein expression levels of glycogen degradation-related factors in cardiac muscle of Brandt's voles.

(a) Representative immunoblots of GYPL in cardiac muscle. (b) Representative polyacrylamide gel of total protein. (c) Relative protein expression of GYPL. Values are mean \pm SD. $n=8$. CON, control group; $\mathrm{CH}$, cool huddling group; CS, cool separated group. Different letters identify statistically significant differences among temperature treatment groups $(P<0.05)$.

\section{References}

1. Gilbert C, McCafferty D, Le Maho Y, Martrette JM, Giroud S, Blanc S, Ancel A: One for all and all for one: the energetic benefits of huddling in endotherms. Biol Rev Camb Philos Soc 2010, 85:545-569.

2. Baum D, Dillard DH, Porte $D$, Jr.: Inhibition of insulin release in infants undergoing deep hypothermic cardiovascular surgery. The New England journal of medicine 1968, 279:1309-1314.

3. Curry DL, Curry KP: Hypothermia and insulin secretion. Endocrinology 1970, 87:750-755.

4. Helman A, Gilbert M, Pfister-Lemaire N, Reach G, Assan R: Glucagon and insulin secretion and their biological activities in hypothermic rats. Endocrinology 1984, 115:1722-1728.

5. Fuhrman GJ, Fuhrman FA: Utilization of glucose by the hypothermic rat. The American journal of physiology 1963, 205:181-183.

6. Popovic V: Physiological characteristics of rats and ground squirrels during prolonged lethargic hypothermia. The American journal of physiology 1960, 199:467-471.

7. Bickford AF, Mottram RF: Glucose metabolism during induced hypothermia in rabbits. Clinical science 1960, 19:345-359.

8. Kelly FE, Nolan JP: The effects of mild induced hypothermia on the myocardium: a systematic review. Anaesthesia 2010, 65:505-515.

9. Polderman $\mathrm{KH}$ : Mechanisms of action, physiological effects, and complications of hypothermia. Crit Care Med 2009, 37:S186-202.

10. Chavez LO, Leon M, Einav S, Varon J: Editor's Choice- Inside the cold heart: A review of therapeutic hypothermia cardioprotection. European Heart Journal-Acute Cardiovascular Care 2017, 6:130-141.

11. Tessier SN, Storey KB: Myocyte enhancer factor-2 and cardiac muscle gene expression during hibernation in thirteen-lined ground squirrels. Gene 2012, 501:8-16.

12. Steffen JM: Glucose, glycogen, and insulin responses in the hypothermic rat. Cryobiology 1988, 25:94-101.

13. Palm DC, Rohwer JM, Hofmeyr JH: Regulation of glycogen synthase from mammalian skeletal muscle--a unifying view of allosteric and covalent regulation. FEBS J 2013, 280:2-27.

14. Zeqiraj E, Sicheri F: Getting a handle on glycogen synthase - Its interaction with glycogenin. Mol Aspects Med 2015, 46:63-69.

15. Wang Z, Jiang SF, Cao J, Liu K, Xu SH, Arfat Y, Guo QL, Chang H, Goswami N, Hinghofer-Szalkay $\mathrm{H}$, Gao YF: Novel findings on ultrastructural protection of skeletal muscle fibers during hibernation of Daurian ground squirrels: Mitochondria, nuclei, cytoskeleton, glycogen. $J$ Cell Physiol 2019, 234:13318-13331.

16. Agius L: Physiological control of liver glycogen metabolism: lessons from novel glycogen 
phosphorylase inhibitors. Mini Rev Med Chem 2010, 10:1175-1187.

17. Mavrokefalos N, Myrianthopoulos V, Chajistamatiou AS, Chrysina ED, Mikros E: Discovery of the Glycogen Phosphorylase-Modulating Activity of a Resveratrol Glucoside by Using a Virtual Screening Protocol Optimized for Solvation Effects. Planta Medica 2015, 81:507-516.

18. Douglas TK, Cooper CE, Withers PC: Avian torpor or alternative thermoregulatory strategies for overwintering? Journal of Experimental Biology 2017, 220:1341-1349.

19. Sukhchuluun G, Zhang XY, Chi QS, Wang DH: Huddling Conserves Energy, Decreases Core Body Temperature, but Increases Activity in Brandt's Voles (Lasiopodomys brandtii). Front Physiol 2018, 9:563.

20. Zhang XY, Sukhchuluun G, Bo TB, Chi QS, Yang JJ, Chen B, Zhang L, Wang DH: Huddling remodels gut microbiota to reduce energy requirements in a small mammal species during cold exposure. Microbiome 2018, 6:103.

21. Jefimow $M$, Glabska $M$, Wojciechowski MS: Social thermoregulation and torpor in the Siberian hamster. J Exp Biol 2011, 214:1100-1108.

22. Wojciechowski MS, Jefimow M, Pinshow B: Heterothermy, and the Energetic Consequences of Huddling in Small Migrating Passerine Birds. Integrative and Comparative Biology 2011, 51:409-418.

23. Kotze J, Bennett NC, Scantlebury M: The energetics of huddling in two species of mole-rat (Rodentia : Bathyergidae). Physiology \& Behavior 2008, 93:215-221.

24. Nunez-Villegas M, Bozinovic F, Sabat P: Interplay between group size, huddling behavior and basal metabolism: an experimental approach in the social degu. Journal of Experimental Biology 2014, 217:997-1002.

25. Namekata S, Geiser F: Effects of nest use, huddling, and torpor on thermal energetics of eastern pygmy-possums. Australian Mammalogy 2009, 31:31-34.

26. Scantlebury M, Bennett NC, Speakman JR, Pillay N, Schradin C: Huddling in groups leads to daily energy savings in free-living African Four-Striped Grass Mice, Rhabdomys pumilio. Functional Ecology 2006, 20:166-173.

27. Nowack J, Geiser F: Friends with benefits: the role of huddling in mixed groups of torpid and normothermic animals. Journal of Experimental Biology 2016, 219:590-596.

28. Sealander JA, Jr.: The Relationship of Nest Protection and Huddling to Survival of Peromyscus at Low Temperature. Ecology, 33:63-71.

29. $\mathrm{X} u \mathrm{D}-\mathrm{L}, \mathrm{X} \cup \mathrm{M}-\mathrm{M}$, Wang $\mathrm{D}-\mathrm{H}$ : Effect of temperature on antioxidant defense and innate immunity in Brandt's voles. Zoological Research 2019, 40:305-316.

30. Wei Y, Zhang J, Xu S, Peng X, Yan X, Li X, Wang H, Chang H, Gao Y: Controllable oxidative stress and tissue specificity in major tissues during the torpor-arousal cycle in hibernating Daurian ground squirrels. Open Biology 2018, 8.

31. Wang Z, Xu J-H, Mou J-J, Kong X-T, Wu M, Xue H-L, Xu L-X: Photoperiod Affects Harderian Gland Morphology and Secretion in Female Cricetulus barabensis: Autophagy, Apoptosis, and Mitochondria. Frontiers in Physiology 2020, 11.

32. Biazik J, Vihinen $H$, Anwar T, Jokitalo E, Eskelinen EL: The versatile electron microscope: an ultrastructural overview of autophagy. Methods 2015, 75:44-53.

33. Reynolds ES: The use of lead citrate at high $\mathrm{pH}$ as an electron-opaque stain in electron microscopy. Journal of Cell Biology 1963, 17:208.

34. Li R, Shen Y: An old method facing a new challenge: re-visiting housekeeping proteins as 
internal reference control for neuroscience research. Life Sci 2013, 92:747-751.

35. Posch A, Kohn J, Oh K, Hammond M, Liu N: V3 stain-free workflow for a practical, convenient, and reliable total protein loading control in western blotting. $J$ Vis Exp 2013:50948.

36. Schaub MC, Brunner UT, Von Schulthess C, Neidhart M, Baumann H: Adaptation of contractile proteins in human heart and skeletal muscles. Biomedica biochimica acta 1989, 48:S306-312.

37. Kanungo S, Wells K, Tribett T, El-Gharbawy A: Glycogen metabolism and glycogen storage disorders. Annals of Translational Medicine 2018, 6:9.

38. Greenberg CC, Jurczak MJ, Danos AM, Brady MJ: Glycogen branches out: new perspectives on the role of glycogen metabolism in the integration of metabolic pathways. Am J Physiol Endocrinol Metab 2006, 291:E1-8.

39. Moniz SC, Islam H, Hazell TJ: Mechanistic and methodological perspectives on the impact of intense interval training on post-exercise metabolism. Scand J Med Sci Sports 2020, 30:638-651.

40. Consitt LA, Dudley C, Saxena G: Impact of Endurance and Resistance Training on Skeletal Muscle Glucose Metabolism in Older Adults. Nutrients 2019, 11. 


\section{Figures}

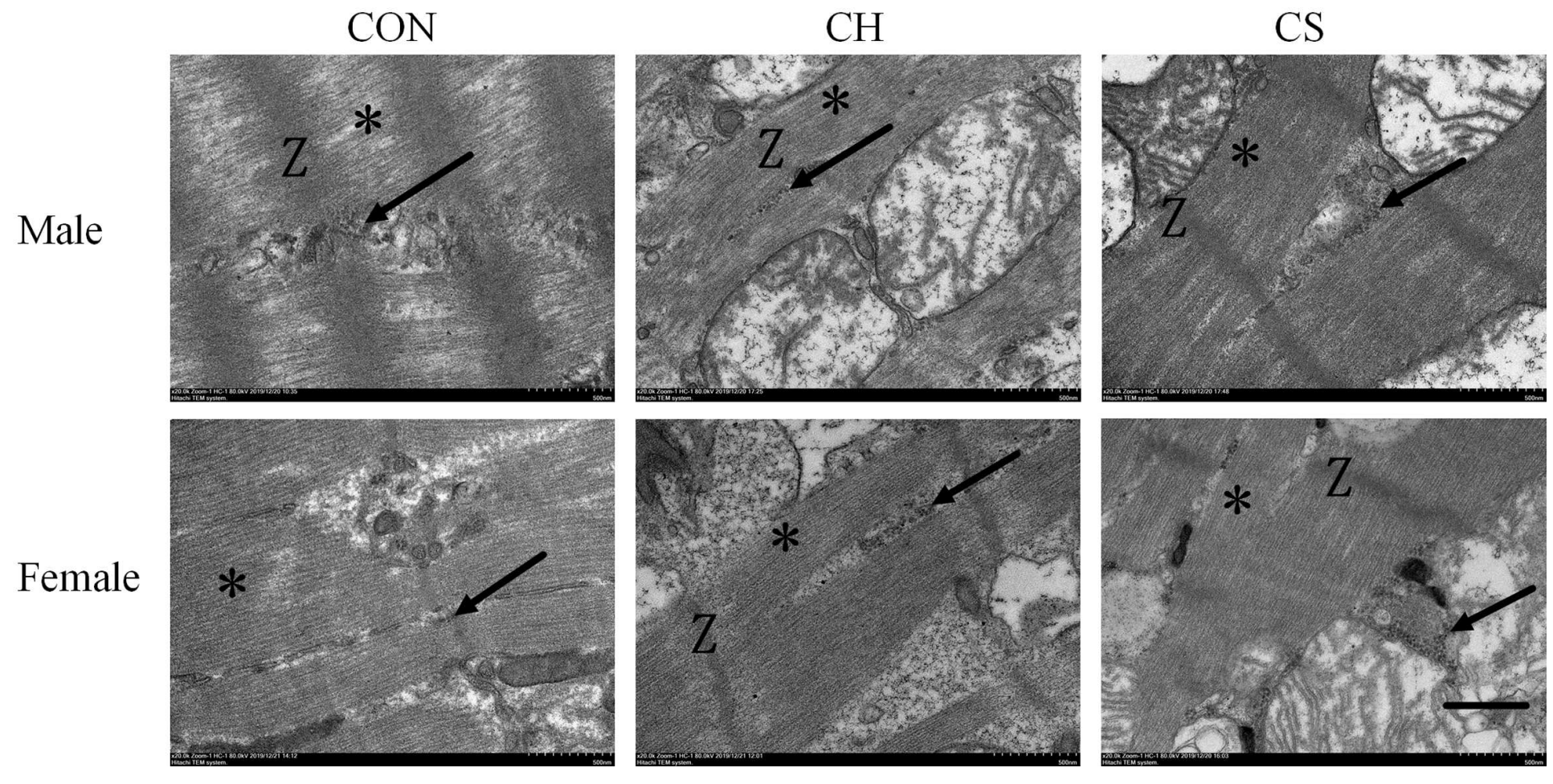

Figure 1

Ultrastructural distribution of myocardial glycogenosomes in Brandt's voles. Arrow indicates glycogenosome. Z-zone (Z) and muscle filaments (see asterisk) are well arranged. Scale bar $=0.5 \mu \mathrm{m}$. 
(a)

$\mathrm{CON}$

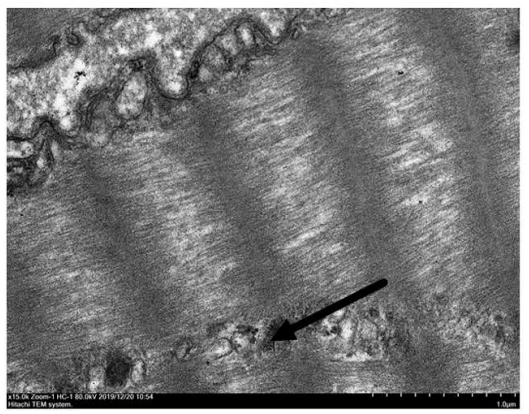

Male

Female

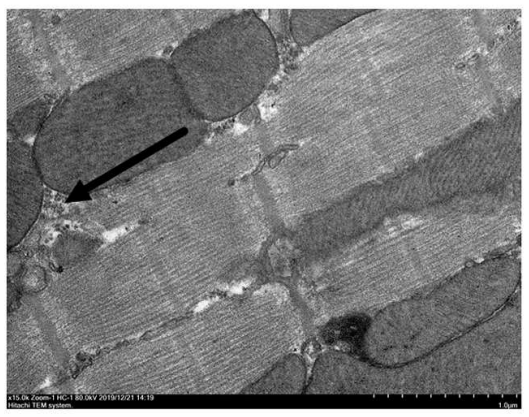

(b)
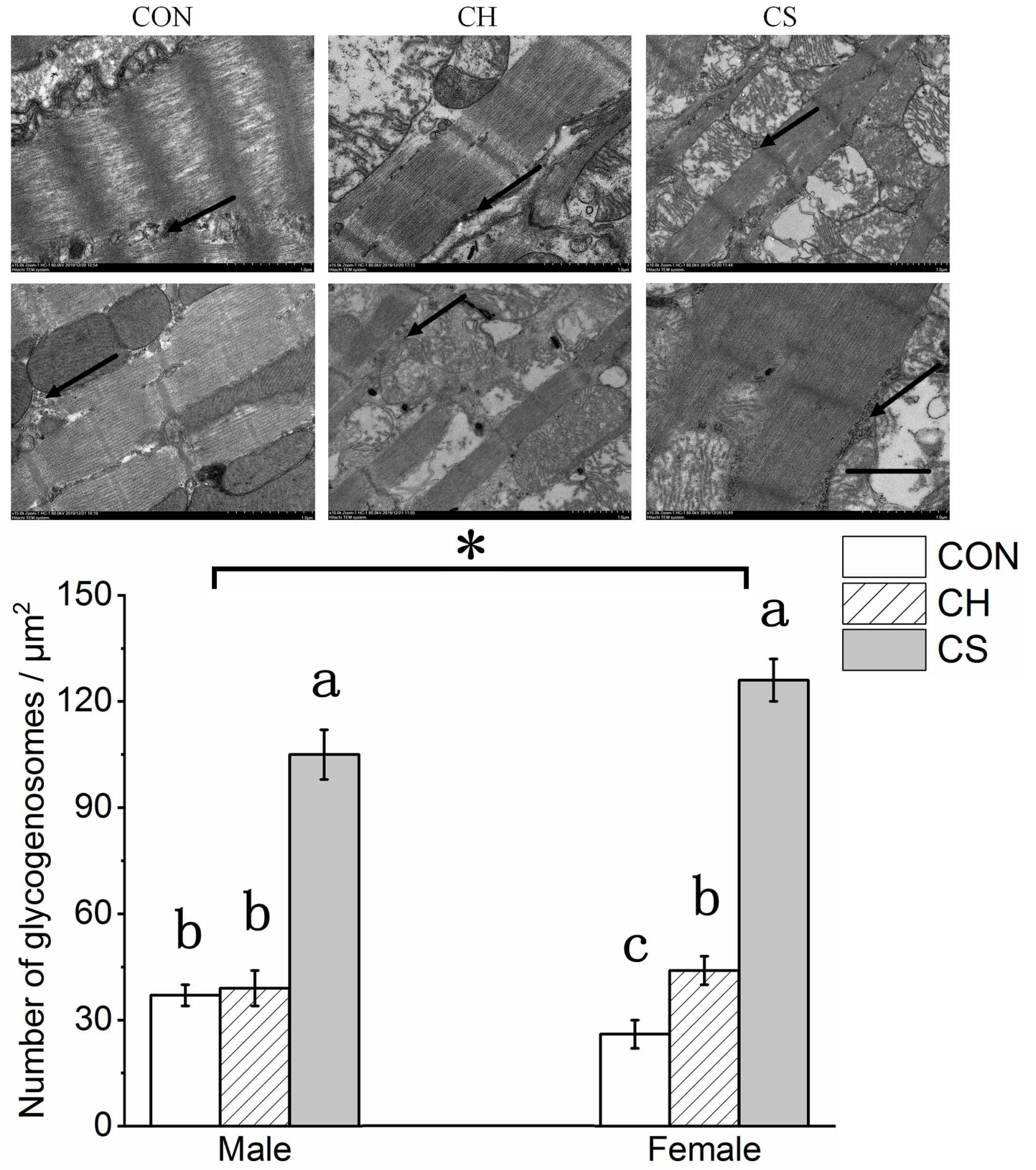

\section{*}

Figure 2

Changes in number of myocardial glycogenosomes in Brandt's voles. (a) Myocardial glycogenosomes in three treatment groups. Arrow indicates glycogenosome. Scale bar $=1 \mu \mathrm{m}$. (b) Bar graph depicting changes in number of glycogenosomes. Values are mean \pm SD. Six figures were analyzed in each sample; eight samples were analyzed in each group. $\mathrm{CON}$, control group; $\mathrm{CH}$, cool huddling group; $\mathrm{CS}$, cool 
separated group. Different letters identify statistically significant differences among temperature treatment groups $(P<0.05)$. *, $P<0.05$ significant differences between males and females.

(a)

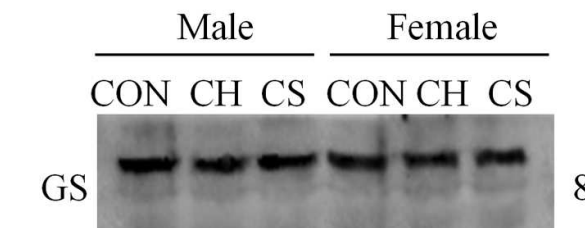

(b)<smiles>C1C[Te]C1</smiles>
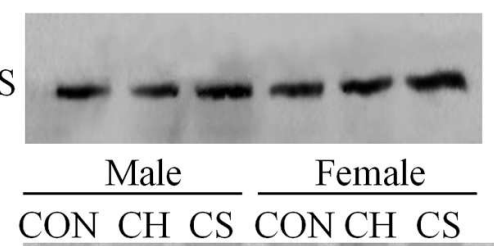

(d)

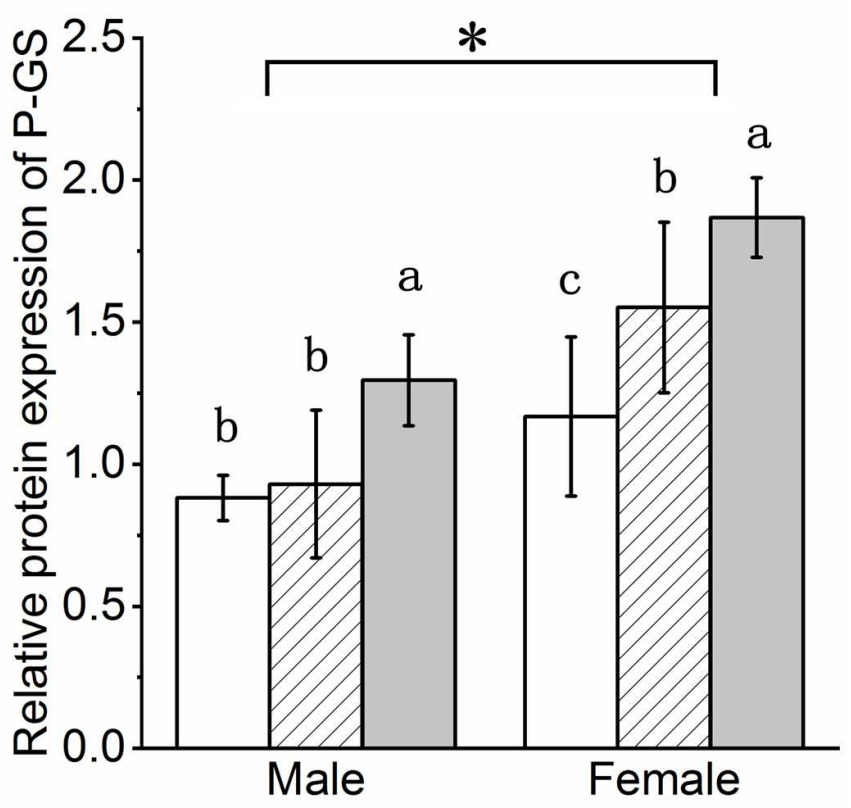

$84 \mathrm{kDa}$

$85 \mathrm{kDa}$ (c)
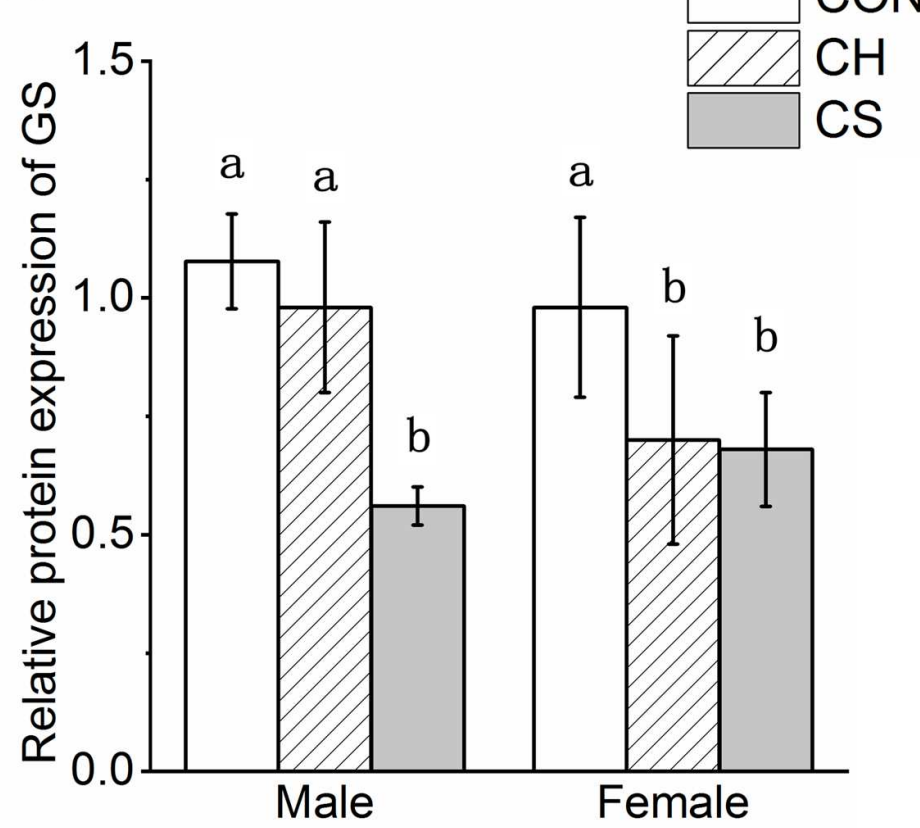

(e)

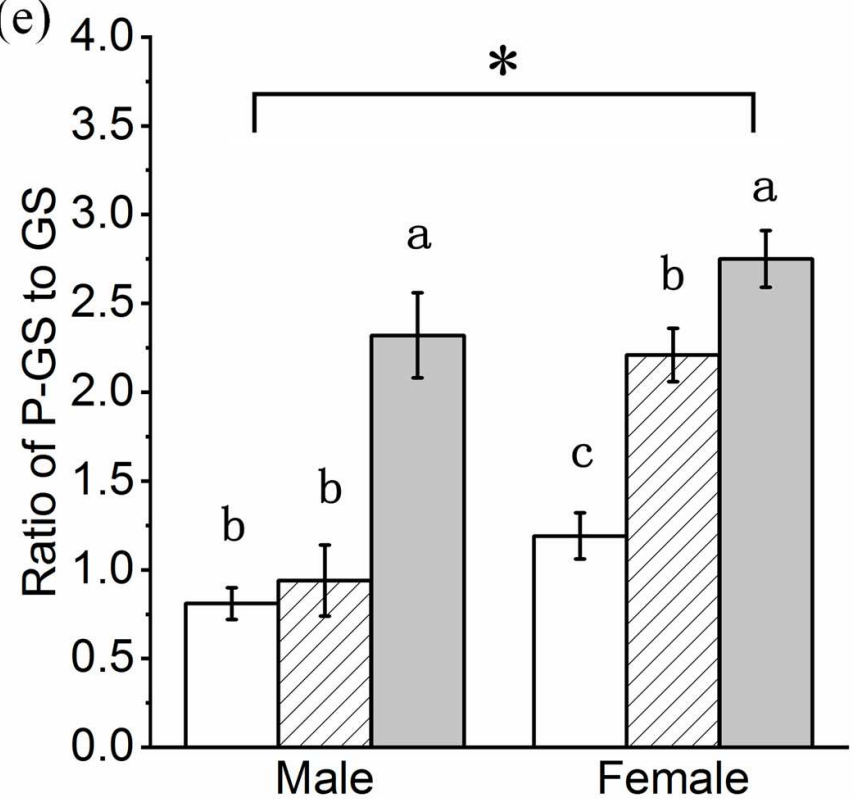

\section{Figure 3}

Changes in protein expression levels of glycogen synthesis-related factors in cardiac (a) Representative immunoblots of GS and P-GS in cardiac muscle. (b) Representative polyacrylamide gel of total protein. (c) Relative protein expression of GS. (d) Relative protein expression of P-GS. (e) Ratio of P-GS to GS. Values are mean $\pm S D . n=8$. CON, control group; $\mathrm{CH}$, cool huddling group; $\mathrm{CS}$, cool separated group. Different letters identify statistically significant differences among temperature treatment groups $(\mathrm{P}<$ $0.05) . *, P<0.05$ significant differences between males and females. 
(a)
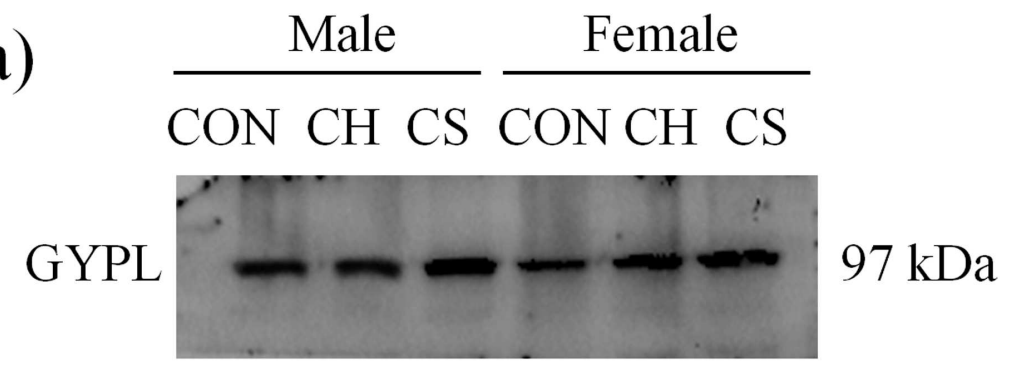

(b)
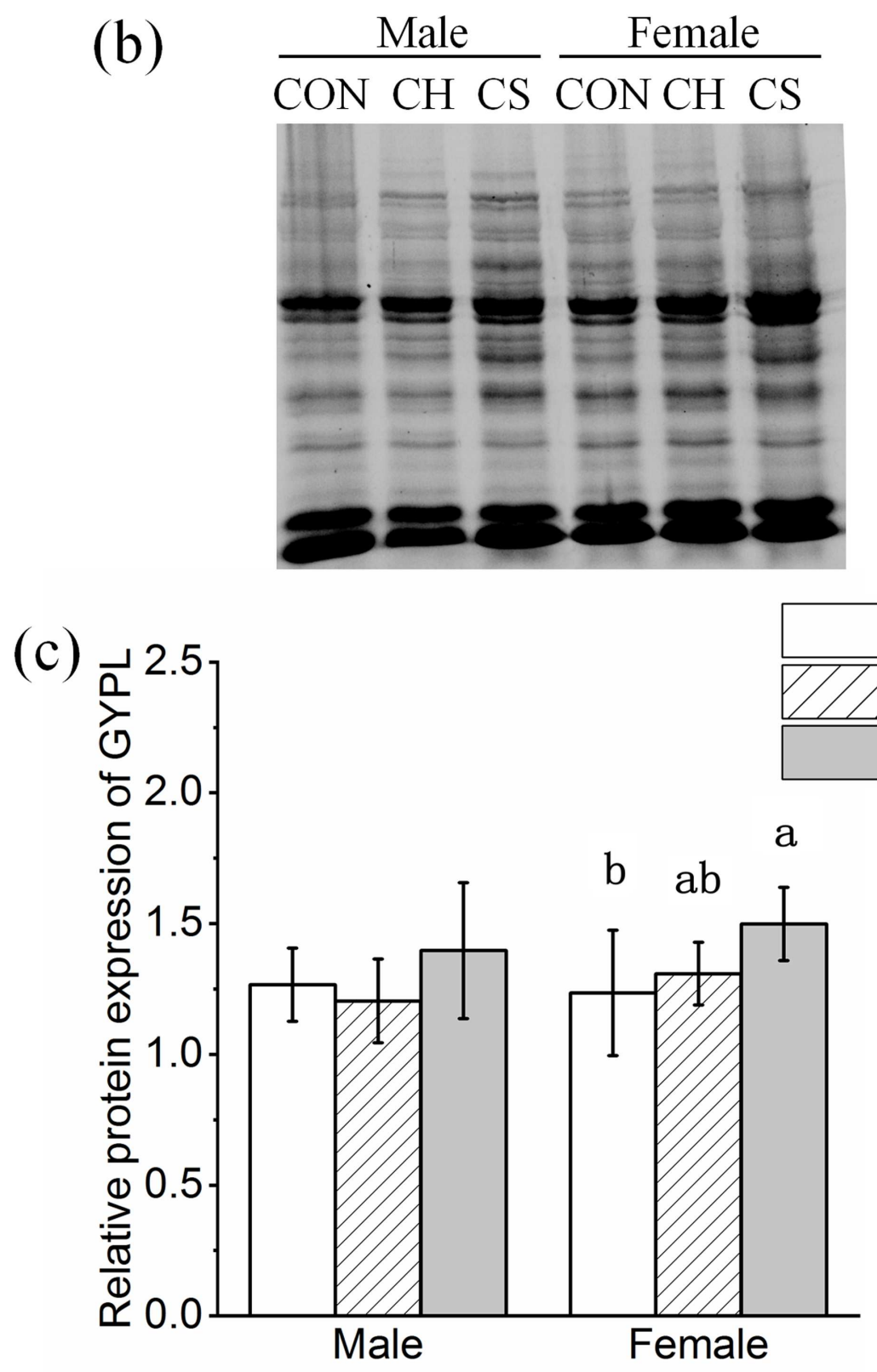

Figure 4

Changes in protein expression levels of glycogen degradation-287 related factors in cardiac muscle of Brandt's voles. (a) Representative immunoblots of GYPL in cardiac muscle. (b) Representative polyacrylamide gel of total protein. (c) Relative protein expression of GYPL. Values are mean \pm SD. $n=8$. $\mathrm{CON}$, control group; $\mathrm{CH}$, cool huddling group; $\mathrm{CS}$, cool separated group. Different letters identify statistically significant differences among temperature treatment groups $(P<0.05)$. 


\section{Supplementary Files}

This is a list of supplementary files associated with this preprint. Click to download.

- supplementary.docx 